infections allow transmission to occur within the facility. Both endemic and epidemic infections occur relatively commonly in nursing homes. The incidence of endemic infections, such as catheter-associated urinary tract infections, lower respiratory infections, and skin infections, is influenced by the debility level of the patients. Calculations of infection rates are influenced by the intensity of surveillance methods at each institution. Many endemic infections are unpreventable.

Epidemic infections account for $10 \%$ to $20 \%$ of nursing home infections. These include clusters of upper or lower respiratory infections, gastroenteritis, diarrhea, and catheter-associated urinary tract infections. Epidemic infections are potentially preventable with sound infection control practices. Special attention must be paid to promote Universal Precautions and give certain patients, such as those with known infection or colonization with Clostridium difficile, methicillin-resistant Staphylococcus aureus, or vancomycin-resistant enterococci, special consideration. The potential for epidemic infections with antibioticresistant organisms is real. In the nursing home setting, strong infection control programs must be in place to monitor the occurrence of institutionally acquired infections and to initiate control strategies to prevent the spread of epidemic infections. Education in infection control issues and attention to employee health are essential to enable staff to care appropriately for today's nursing home population and to prepare them for the even more complicated patients who will be cared for in this type of setting in future.

FROM: Garibaldi RA. Residential care and the elderly: the burden of infection.J Hosp Infect 1999;43(suppl):S9-S18.

\section{Survival of Enterococci and Staphylococci on Hospital Fabrics and Plastic}

The transfer of gram-positive bacteria, particularly methicillin-resistant Staphylococcus aureus (MRSA) and vancomycin-resistant enterococci (VRE), among patients is a growing concern. One aspect of bacterial transfer is the ability of the microorganism to survive on various common hospital surfaces. Neely and Maley from the Shriners Hospitals for Children, University of Cincinnati College of Medicine, conducted a study to determine the survival of 22 gram-positive bacteria (vancomycin-sensitive and -resistant enterococci and methicillin-sensitive and -resistant staphylococci) on five common hospital materials: smooth $100 \%$ cotton (clothing), 100\% cotton terry (towels), 60\% cotton$40 \%$ polyester blend (scrub suits and laboratory coats), $100 \%$ polyester (privacy drapes), and 100\% polypropylene plastic (splash aprons). Swatches were inoculated with $10^{4}$ to $10^{5}$ colony-forming units of a microorganism, assayed daily by placing the swatches in nutritive media, and examined for growth after 48 hours. All isolates survived for at least 1 day, and some survived for more than 90 days on various materials. Smaller inocula $\left(10^{2}\right)$ survived for shorter times, but still generally for days.
The authors concluded that antibiotic sensitivity had no consistent effect on survival. The long survival of these bacteria, including MRSA and VRE, on commonly used hospital fabrics, underscores the need for meticulous contact control procedures and careful disinfection to limit the spread of these bacteria.

Editorial note: The authors have confirmed that gram-positive bacteria such as enterococci and staphylococci are more hardy than most gram-negative bacteria and that antibiotic resistance does not affect survival in the environment. There have been a number of studies to determine the factors associated with the transmission of MRSA and VRE among hospitalized patients. Clearly, cross-contamination via hands of healthcare personnel has been found to be a major risk factor for transmission. Although bacteria have been shown to survive on fabrics, they have not been directly associated with transmission of VRE or MRSA.

FROM: Neely AN, Maley MP. Survival of enterococci and staphylococci on hospital fabrics and plastic. J Clin Microbiol 2000;38:724-726.

\section{Vancomycin Treatment and Hospital- Acquired VRE: A Meta-analysis}

The association between vancomycin hydrochloride treatment and vancomycin-resistant enterococci (VRE) has been investigated in numerous studies with variable results. Carmeli and colleagues from Beth Israel Deaconess Medical Center and Harvard Medical School, Boston, conducted a meta-analysis to estimate the magnitude of the association between vancomycin treatment and individual risk of VRE and to identify study characteristics that accounted for heterogeneity in study results.

Studies were identified using MEDLINE, searching the index with terms "Enterococcus," "Enterococcus faecalis," or "Enterococcus faecium" and "vancomycin," "drug resistance," "drug resistance, microbial," or "drug resistance, multiple or risk factors." Reports from conferences and reference lists of recent reviews were used. A total of 420 published reports and 98 conference reports were reviewed; 20 studies described in 15 published reports were included in the analysis. They recorded study period, hospital setting, case and control definitions, length of hospital stay, method of adjustment for differences in length of stay, and data on treatment with vancomycin.

The odds ratio (OR) of vancomycin treatment provided the measure of association analyzed. A randomeffects model was used to estimate the pooled OR. When results from all 20 studies were combined, the pooled $O R$ was 4.5 (95\% confidence interval $\left.\left[\mathrm{CI}_{95}\right], 3.0-6.9\right)$, but the test for heterogeneity was highly significant $(P<.001)$. The 5 studies that used patients with vancomycin-susceptible enterococci as controls found a stronger association (pooled OR, 10.7; $\mathrm{CI}_{95}, 4.8-23.8$ ) than the 15 studies that used controls who had no VRE isolated (pooled OR, 2.7; $\mathrm{CI}_{95}, 2.0-3.8$ ). After restricting the analysis to the latter 